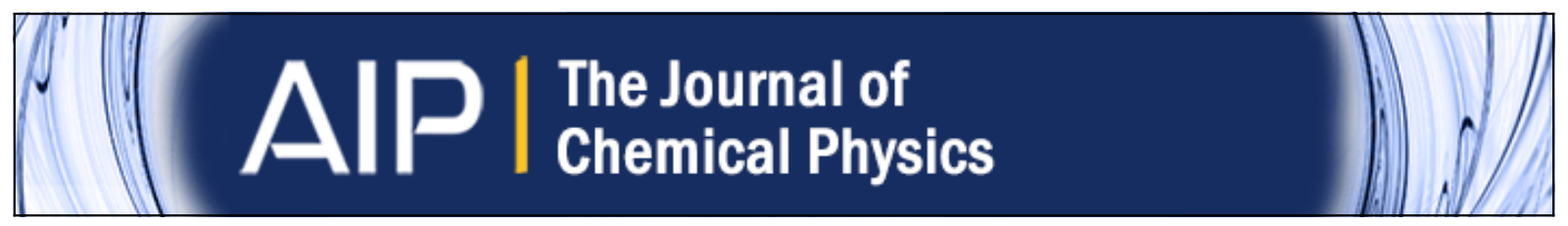

\title{
Structure and dynamics of liquid methanol confined within functionalized silica nanopores
}

M. Dolores Elola, Javier Rodriguez, and Daniel Laria

Citation: The Journal of Chemical Physics 133, 154707 (2010); doi: 10.1063/1.3503886

View online: http://dx.doi.org/10.1063/1.3503886

View Table of Contents: http://scitation.aip.org/content/aip/journal/jcp/133/15?ver=pdfcov

Published by the AIP Publishing

\section{Articles you may be interested in}

Asymmetric orientation of toluene molecules at oil-silica interfaces

J. Chem. Phys. 137, 064703 (2012); 10.1063/1.4742696

Reorientation dynamics of nanoconfined water: Power-law decay, hydrogen-bond jumps, and test of a two-state model

J. Chem. Phys. 136, 044513 (2012); 10.1063/1.3679404

Structure and dynamics of water confined in silica nanopores

J. Chem. Phys. 135, 174709 (2011); 10.1063/1.3657408

Aqueous electrolytes confined within functionalized silica nanopores

J. Chem. Phys. 135, 104503 (2011); 10.1063/1.3632050

Structural studies of water in hydrophilic and hydrophobic mesoporous silicas: An x-ray and neutron diffraction study at $297 \mathrm{~K}$

J. Chem. Phys. 134, 064509 (2011); 10.1063/1.3530584

How can you REACH $100 \%$

of researchers at the Top 100

Physical Sciences Universities?

With The Journal of Chemical Physics.

AIP $\mid \begin{aligned} & \text { The Journal of } \\ & \text { Chemical Physics }\end{aligned}$

THERE'S POWER IN NUMBERS. Reach the world with AIP Publishing.

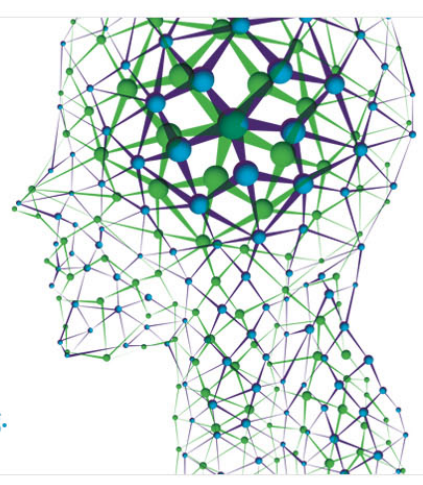




\title{
Structure and dynamics of liquid methanol confined within functionalized silica nanopores
}

\author{
M. Dolores Elola, ${ }^{1, a)}$ Javier Rodriguez, ${ }^{1,2}$ and Daniel Laria ${ }^{1,3}$ \\ ${ }^{1}$ Departamento de Física, Comisión Nacional de Energía Atómica, Avenida Libertador 8250, 1429 Buenos \\ Aires, Argentina \\ ${ }^{2}$ ECyT, UNSAM, Martín de Irigoyen 3100, 1650 San Martín, Provincia de Buenos Aires, Argentina \\ ${ }^{3}$ Departamento de Química Inorgánica Analítica y Química-Física e INQUIMAE, Facultad de Ciencias \\ Exactas y Naturales, Universidad de Buenos Aires, Ciudad Universitaria, Pabellón II, 1428 Buenos \\ Aires, Argentina
}

(Received 10 August 2010; accepted 29 September 2010; published online 21 October 2010)

\begin{abstract}
Molecular dynamics simulations have been carried out to investigate the structure and dynamics of liquid methanol confined in $3.3 \mathrm{~nm}$ diameter cylindrical silica pores. Three cavities differing in the characteristics of the functional groups at their walls have been examined: (i) smooth hydrophobic pores in which dispersive forces prevail, (ii) hydrophilic cavities with surfaces covered by polar silanol groups, and (iii) a much more rugged pore in which $60 \%$ of the previous interfacial hydroxyl groups were replaced by the bulkier trimethylsilyl ones. Confinement promotes a considerable structure at the vicinity of the pore walls which is enhanced in the case of hydroxylated surfaces. Moreover, in the presence of the trimethylsilyl groups, the propagation of this interface-induced spatial ordering extends down to the central region of the pore. Concerning the dynamical modes, we observed an overall slowdown in both the translational and rotational motions. An analysis of these mobilities from a local perspective shows that the largest retardations operate at the vicinity of the interfaces. The gross features of the rotational dynamics were analyzed in terms of contributions arising from bulk and surface states. Compared to the bulk dynamical behavior, the characteristic timescales associated with the rotational motions show the most dramatic increments. A dynamical analysis of hydrogen bond formation and breaking processes is also included. (C) 2010 American Institute of Physics. [doi:10.1063/1.3503886]
\end{abstract}

\section{INTRODUCTION}

Fluids confined within environments with nanometric dimensions are ubiquitous throughout nature. An appropriate physical interpretation of the modifications operated in these trapped phases, compared to what is found in more usual bulk-like scenarios, is essential to understand how finite size effects influence the behavior of a confined liquid. The list of examples spans from a wide variety of technological applications, such as crevice corrosion processes in boilers, ${ }^{1}$ the recovery of oil trapped within porous rocks, ${ }^{2}$ the characteristic of steams within geothermal reservoirs, ${ }^{3}$ problems with fundamental biological relevance such as the solvation at the surface of proteins, ${ }^{4}$ or the hydration in highly concentrated sugar solutions. ${ }^{5}$

Nanoporous silicate glasses have proven to be the excellent media for studying confinement effects upon the structure and dynamics of trapped liquid phases. A variety of experimental techniques has been recently implemented to analyze these issues including NMR, ${ }^{6-10}$ Rayleigh $^{11,12}$ and neutron ${ }^{13,14}$ scattering, and several spectroscopic methods, such as Raman, ${ }^{15-17}$ dielectric, ${ }^{18-21}$ and optical Kerr effect. ${ }^{22-26}$ New methods in chemical synthesis allow subnanometric control of the pore linear dimensions and, at the

\footnotetext{
a) Author to whom correspondence should be addressed. Electronic mail: DoloresElola@gmail.com.
}

same time, an accurate "tuning" of the characteristics of the interactions prevailing between the solid walls and the trapped liquid phases. In the case of silica substrates, this gives rise to a wide variety of environments, normally described in terms of their hydrophobic and/or hydrophilic characteristics.

In the present paper, we will present the results from molecular dynamics (MD) experiments performed on model systems of methanol $(\mathrm{MeOH})$ trapped within different cylindrical silica pores with linear dimensions close to $3 \mathrm{~nm}$. This particular choice of pore size responds to two different reasons. (i) First, it is in this range of pore diameters where one starts perceiving distinctions between structural and dynamical behaviors corresponding to "bulk-like" states at the center of the pore and "adsorbed" ones in regions closer to the pore walls. (ii) Second, the numbers of molecules contributing to these states are still comparable. This, in principle, would make it possible to "dissect" the overall characteristics into separate contributions with comparable weights.

On the other hand, liquid methanol also represents an interesting choice for the trapped fluid phase. Although its microscopic structure is dictated to a large extent by the architecture of hydrogen bonds (HBs), the presence of a methyl group introduces a hydrophobic character that makes the methanol "amphiphilic," a characteristic that is absent, for example, in the most common case of liquid water. As a consequence, the dimensionality of the prevailing intermo- 
lecular spatial correlations suffers from important modifications which is normally cast in terms of chains of $\mathrm{HBs}^{27,28}$ instead of the usual three-dimensional network that prevails in liquid water.

The structure of methanol confined within cylindrical pores of MCM-41 has been recently investigated by neutron scattering experiments, ${ }^{13,14}$ large-angle x-ray scattering ${ }^{29}$ experiments, and Monte Carlo simulations as well. ${ }^{30}$ Both experimental and simulation results confirm that the presence of surface silanol groups at the pore walls affects the spatial correlations between the interfacial $\mathrm{MeOH}$ molecules in a sensible fashion. From the dynamical side, these modifications also promote important slowdowns in the dielectric response of the fluid. ${ }^{14}$ Similar conclusions have been obtained from time-resolved fluorescence spectroscopy measurements of the solvation dynamics of a probe coumarin 153 immersed in $\mathrm{MeOH}$ within silica nanochannels. ${ }^{31}$

In a related context, MD experiments have also been recently performed on methanol and water/methanol mixtures trapped within single wall carbon nanotubes. ${ }^{32-34}$ In addition to the above mentioned retardations, simulation results have revealed that the spatial inhomogeneities also give rise to local modifications of the translational and rotational modes of the trapped molecules. From experimental grounds, these dynamical inhomogeneities have also been confirmed by Fourkas and collaborators. ${ }^{26,35,36}$ Results from ultrafast optical Kerr effect spectroscopy performed on carbon disulfide and acetonitrile confined in silica nanopores are consistent with the existence of a layer of molecules at the pore surfaces which experiences a higher viscosity, compared to the one prevailing at central regions of the pore.

Motivated by the large body of previous results and as an extension of our recent study in which we analyzed the behavior of confined polar mixtures in functionalized silica nanopores, ${ }^{37}$ we will present the results describing the behavior of trapped $\mathrm{MeOH}$ in three different silica pores with nanometric dimensions. The emphasis will be focused on the effects of the different liquid-wall interactions upon the resulting structure and different dynamical modes of the trapped liquid phase.

The paper is organized as follows. In Sec. II, a description of the model and the technical details of the simulations are presented. In Sec. III, we present and discuss the results obtained for the structural and dynamical properties of confined methanol. A summary of the main conclusions is presented in Sec. IV.

\section{MODELS AND COMPUTATIONAL DETAILS}

Confinement effects were examined in fully periodic systems comprising cylindrical silica pores filled with liquid methanol. To build up the solid substrate, we followed closely the procedure described in previous publications, ${ }^{37-42}$ so we will briefly comment on the more important aspects of the scheme and refer the interested reader to those papers for additional details. We started by considering a sample of fused $\mathrm{SiO}_{2}$ within a simulation box of linear dimensions $L_{x}$ $=L_{y}=51 \AA, L_{z}=33 \AA$, equilibrated at temperatures close to $T \sim 8000 \mathrm{~K}$. After immobilizing a central cylindrical section

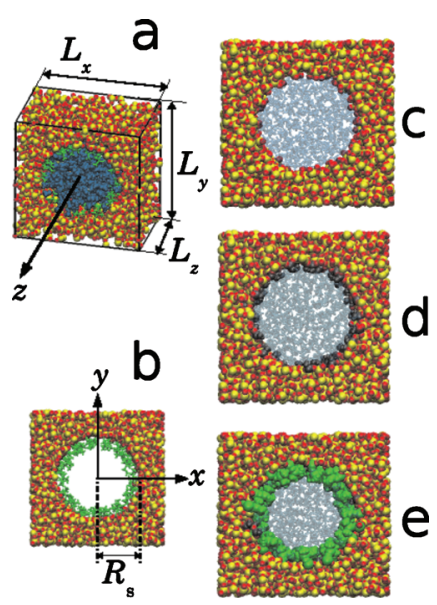

FIG. 1. Left panels (a) and (b) show the orientation of the simulation box relative to the laboratory axes; $R_{s}$ is the radius of the silica pore. On right panel, snapshots of typical configurations for (c) HOC, (d) HIC, and (e) RHOC simulated silica nanopores are shown. $\mathrm{SiO}_{2}$ groups are depicted in red and yellow, methanol in blue, silanol $(\mathrm{SiOH})$ in gray [panel (d)], and trimethylsilyl groups in green [panel (e)].

of radius $R_{s}=16.5 \AA$ along the $z$-axis, the surrounding portion was allowed to cool down to ambient conditions by multiple rescaling of the atomic velocities. The central portion of the sample was then removed, leaving a cylindrical pore of radius $R_{s}$, across the silica slab with dynamical characteristics similar to a solid [see Figs. 1(a) and 1(b)].

Three pores differing in the functionalization of the cavity walls were investigated. (i) By simply canceling all Coulomb interactions, we first analyzed hydrophobic cavities (HOC), in which only dispersive, i.e., Lennard-Jones, forces prevail. Note that these environments only retain the gross features akin to hydrophobic cavities and, in its construction, no efforts were put in reproducing experimental protocols normally implemented to control the degree of hydrophobicity within silica pores. (ii) Second, we also considered hydrophilic cavities (HIC) in which unsaturated oxygens at the internal walls were hydroxylated, yielding a density of $\mathrm{SiOH}$ groups of $\sim 3 \mathrm{~nm}^{-2}$. This value is in reasonable agreement with the data obtained from gas adsorption experiments. ${ }^{31}$ (iii) Finally, we analyzed the case of more rugged hydrophobic-like cavities (RHOC) in which $60 \%$ of the previous silanol groups were replaced by bulkier $\mathrm{O}-\mathrm{Si}-\left(\mathrm{CH}_{3}\right)_{3}$ trimethylsilyl (TMS) groups. In the three cases, after the annealing procedure, all atoms in the block remained immobile, with the exception of the hydrogen ones in the silanol groups and those comprising the TMS moieties. Figures 1(c)-1(e) show snapshots of the typical configurations for the three classes of silica pores investigated.

The subsequent stage involved the incorporation of the liquid phase within the different pores. To do so, we enlarged the simulation box along the $z$-axis up to $80 \AA$ by juxtaposing the solid blocks to an adjacent bulk-like reservoir with linear dimensions $L_{x}=L_{y}=51 \AA, L_{z}=47 \AA$, filled with $\sim 1800$ methanol molecules. From then on, the liquid phase was allowed to reequilibrate by permeating through the pore for about 500 ps. During this period, the length of the simulation box along the $z$-axis was adjusted to set the local density at the center of the reservoir to the bulk value at ambient 
TABLE I. Number of molecules of each species within the silica cavities.

\begin{tabular}{lcccc}
\hline \hline & & \multicolumn{3}{c}{ Cavity type } \\
\cline { 3 - 5 } & Species & HOC & HIC & RHOC \\
\hline$N_{\mathrm{MeOH}}$ & $\mathrm{CH}_{3} \mathrm{OH}$ & 373 & 390 & 300 \\
$N_{\mathrm{SiOH}}$ & $\mathrm{SiOH}$ & $\ldots$ & 86 & 32 \\
$N_{\mathrm{TMS}}$ & $\mathrm{SiOSi}\left(\mathrm{CH}_{3}\right)_{3}$ & $\ldots$ & $\ldots$ & 55 \\
\hline
\end{tabular}

conditions, $\rho_{\text {bulk }}=0.0148 \AA^{-3}$. Finally, the lateral reservoir was removed bringing the linear dimension along the $z$-axis of the periodic system back to $33 \AA$. The final numbers of $\mathrm{MeOH}$ molecules in each system are listed in Table I. As we will show in Sec. III A, with this procedure, the local density of $\mathrm{MeOH}$ at the center of the pore was found to be in reasonable agreement with the above mentioned bulk value, a fact that has been reported in previous experimental ${ }^{13}$ and simulations ${ }^{30}$ studies.

To model interactions involving methanol molecules, we adopted the CHARMM27 force field. ${ }^{43}$ Within this parametrization, the molecule comprises six sites with intramolecular interactions that include stretching, bending, and dihedral contributions. The inter- and intramolecular potential parameters employed for silica, silanol, and trimethylsilyl, and the partial charges assigned to each atomic site can be found in our previous work. ${ }^{37}$

The trajectories were generated using the NAMD package $^{44}$ which corresponded to the microcanonical ensemble. The particle mesh Ewald method was used to handle long-range Coulomb forces. ${ }^{45,46}$ The equations of motion were integrated using a multiple time step integration scheme with a time step of 1 fs. After filling the different silica cavities with methanol, we equilibrated the systems for $\sim 0.5 \mathrm{~ns}$ at $T=298 \mathrm{~K}$. After this initial period, configurations were saved at time intervals of 0.25 ps along $\sim 6-10$ ns runs to collect appropriate statistics.

\section{RESULTS AND DISCUSSION}

\section{A. Structure}

The different pores represent nonuniform confining environments. To facilitate the description that follows, it will be convenient to define a local coordinate system whose origin is at the center-of-mass of the solid phase. We start our analysis by examining the local density field of $\mathrm{MeOH}$ along the radial direction within the pore,

$$
\rho(r)=\frac{1}{2 \pi r L_{z}} \sum_{i}\left\langle\delta\left(r-R_{i}\right)\right\rangle,
$$

where $R_{i}=\sqrt{X_{i}^{2}+Y_{i}^{2}}$ denotes the distance between the centerof-mass of the $i$ th methanol molecule and the axis of the cavity [see Fig. 1(b)]. The radial density profiles normalized by the bulk density are displayed in Fig. 2. In the HOC and HIC cavities, one observes central regions of radii $r$ $\lesssim 11 \AA$ and $r \lesssim 8 \AA$, respectively, where the local density profiles remain practically structureless at $\rho(r) \sim \rho_{\text {bulk }}$. As we approach the silica wall, fluctuations can be clearly perceived: in the HOC case, one observes a local maximum of

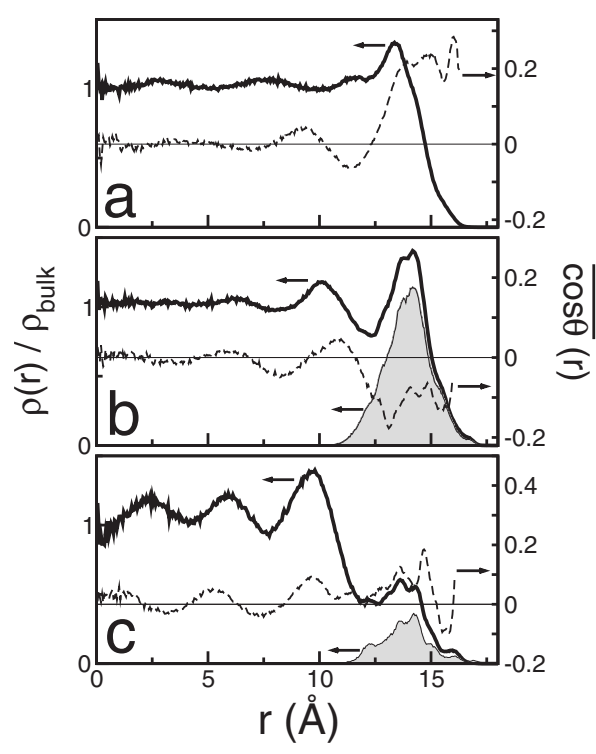

FIG. 2. Solid lines, left axis: local density profiles of methanol along the radial direction. Dashed lines, right axis: angular correlations where $\cos \theta$ corresponds to the projection of $\mathbf{r}_{\mathrm{C}}-\mathbf{r}_{\mathrm{O}}(\mathrm{O} \rightarrow \mathrm{C}$ vector of methanol $)$ along the radial direction of the pore as a function of $r$. Panels (a)-(c) correspond to HOC, HIC, and RHOC silica pore cavities, respectively. The shaded areas in panels (b) and (c) indicate the contribution to the density from methanol molecules $\mathrm{H}$-bonded to surface silanols.

height 1.3 at $r=13 \AA$, whereas in hydrophilic pores, the position of this maximum shifts closer to the silica wall, $r$ $\sim 14 \AA$. In addition, hydroxylation promotes a larger extent of liquid structure toward the interior of the pore. This is revealed by the presence of a secondary maximum of smaller amplitude at $r \sim 10 \AA$. Modifications are even more marked in RHOCs, where the profile of $\rho(r)$ exhibits a much lower surface peak and three solvent layers at regular intervals of about 3.8-4 $\AA$, a lengthscale comparable to the molecular size of a MeOH molecule. Moreover, in this case, the propagation of this interface-induced spatial ordering extends deeper into the liquid phase, practically down to the central portion of the pore.

The integration of the density profile peaks near the silica interface (i.e., $r \geq 12 \AA$ ) shows that $38 \%$ and $41 \%$ of the confined $\mathrm{MeOH}$ molecules lie at the interface in HOC and HIC cases, respectively. Within RHOCs, the presence of the highly rugose interface comprising bulkier TMS groups reduces the effective $\mathrm{MeOH}$ packing, bringing the previous percentage down to $24 \%$.

In Fig. 2, the shaded areas correspond to contributions to the density arising from $\mathrm{MeOH}$ molecules $\mathrm{H}$-bonded to surface silanol groups. Following previous works, ${ }^{5,47}$ our definition of a HB resorted on a geometrical criterion based on a maximum threshold $\mathrm{O} \cdots \mathrm{O}$ distance of $3.4 \AA$ and a maximum $\mathrm{O} \cdots \mathrm{O}-\mathrm{H}$ angle of $30^{\circ}$. The integration of the latter density profiles shows that $80 \%$ of the $\mathrm{MeOH}$ molecules at the contact layer are engaged in $\mathrm{H}$-bonds with $\mathrm{Si}-\mathrm{OH}$ groups in the HIC case, whereas only $48 \%$ of the interfacial $\mathrm{MeOH}$ molecule participates in $\mathrm{H}$-bonding with $\mathrm{Si}-\mathrm{OH}$ groups in the RHOC pore. Still, these percentages would indicate that practically all silanol groups at the inner silica pore surfaces are involved in $\mathrm{H}$-bonds with nearby $\mathrm{MeOH}$ molecules. 

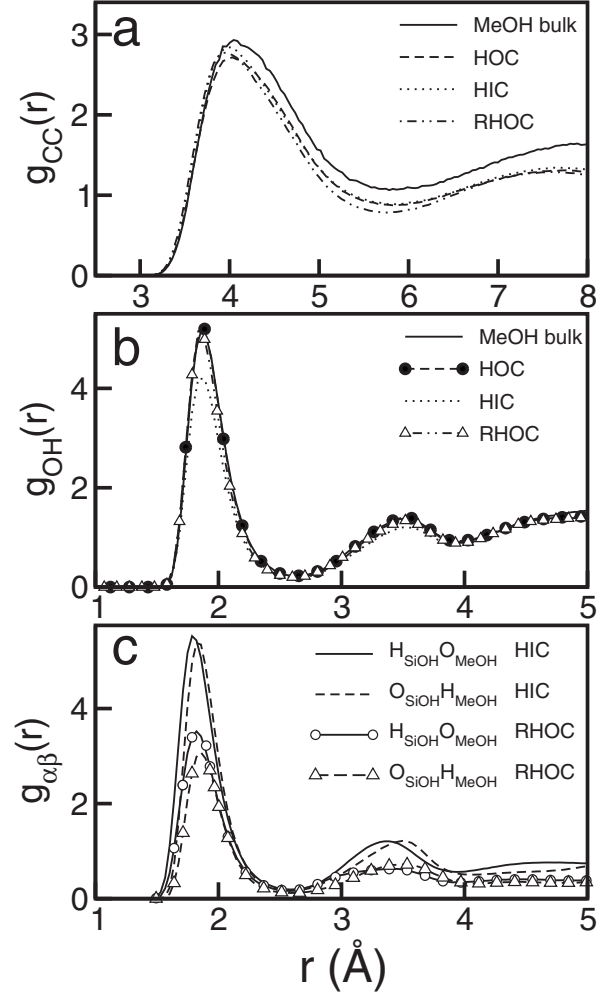

FIG. 3. Radial distribution functions between pairs of methanol molecules [panels (a) and (b)] and between methanol and silanol sites [panel (c)].

Concerning orientational correlations induced by the confinement, we found it convenient to focus our analysis on correlation functions of the type

$$
\overline{\cos \theta}(r)=\frac{\sum_{i}\left\langle\delta\left(\cos \theta-\cos \theta_{i}\right) \delta\left(r-R_{i}\right)\right\rangle}{\sum_{i}\left\langle\delta\left(r-R_{i}\right)\right\rangle},
$$

where $\cos \theta_{i}$ corresponds to the projection of the unit vector along the $\mathbf{r}_{i}^{\mathrm{C}}-\mathbf{r}_{i}^{\mathrm{O}}$ direction onto the radial direction of the pore. Here, $\mathbf{r}_{i}^{\alpha}$ represents the coordinate of the $\alpha$ site in the $i$ th molecule. Results for $\overline{\cos \theta}(r)$ are also included in Fig. 2 (right axes). The three profiles show no relevant structure at the central parts of the pores, say, for $r \leqq 8-10 \AA$. At the vicinity of the HOC walls, the positive deviation of the curve reveals that methanol molecules expose their methyl groups toward the solid phase. Contrasting, in HICs, the prevailing orientations look reversed, suggesting liquid-substrate intermolecular hydrogen-bond connectivity of the type $\mathrm{Me}-\mathrm{OH} \cdots \mathrm{OH}-\mathrm{Si}$ and/or Me-HO $\cdots \mathrm{HO}-\mathrm{Si}$. Finally, in RHOCs, there is no clear sign of a prevailing orientation at the interface, most likely prevented by larger extent of topological disorder introduced by the presence of the mobile and bulkier TMS groups, scattered all along the solid interface.

The local structure around $\mathrm{MeOH}$ molecules was analyzed in terms of radial distribution functions (RDFs) of the type

$$
g_{\alpha \beta}(r)=\frac{1}{4 \pi r^{2} N_{\alpha}} \sum_{i}^{N_{\alpha}} \sum_{j \neq i}^{N_{\beta}}\left\langle\delta\left(r-r_{i j}^{\alpha} \gamma\right)\right\rangle,
$$

where $N_{\alpha}$ represents the number of sites of species $\alpha$ and $r_{i j}^{\alpha \gamma}=\left|\mathbf{r}_{i}^{\alpha}-\mathbf{r}_{j}^{\beta}\right|$. In the three panels of Fig. 3, we present the
RDFs for a few relevant site-pairs. To analyze the overall features of the solvent-solvent spatial correlations, it will instructive to start by analyzing C-C RDFs [Fig. 3(a)]. Note that the positions and the areas under the main peaks centered at $r \sim 4 \AA$ in the bulk and in the pores look qualitatively similar, with an average coordination number close to 12. Of course, the confinement leads to somewhat less prominent peaks and a gradual decrease of the asymptotic long- $r$ behavior (due to the lack of spherical symmetry within the pores). Modifications operated in the hydrogen bond structure of the liquid can be better gauged from the characteristics of the different RDFs corresponding to $\mathrm{O}-\mathrm{H}$ pairs. In the middle panel, we present the results for spatial correlations involving solvent sites exclusively. The resulting areas under the main peaks centered at $r=1.9 \AA$ add up 0.96 for the HOC, 0.78 for the HIC, and 0.91 for the ROHC, whereas in the bulk (also shown in the figure) the corresponding area is 0.97 . These numbers confirm that compared to the bulk situation, H-bonding at the central regions of the pores-which, in all cases, represent the major contribution to the pair correlations-is preserved with minor alterations, i.e., most molecules exhibit single-donor-single-acceptor characteristics.

Modifications in the intermolecular connectivity in the outer regions of the pores can be examined from the characteristics of $g_{\alpha \gamma}(r)$, with $\alpha=\mathrm{O}, \mathrm{H}$ belonging to silanol groups. Results for these site-site correlations appear at the bottom panel of Fig. 3. The magnitudes of the main peaks reveal that in HIC, most of the silanol groups exhibit dual donoracceptor characteristics operated via $\mathrm{MeOH}$ bridging nearby molecules. 95\% of them act as H-bond-acceptors from nearby $\mathrm{MeOH}$ molecules and $88 \%$ act as H-bond-donors. In RHOCs, the previous percentages dropped considerably (down to 65\% for H-bond-acceptor groups and to 54\% for $\mathrm{H}$-bond-donor ones), indicating that the presence of TMS groups precludes $\mathrm{MeOH}$ bridging structures between nearby silanol groups.

A more clear insight about the characteristics of HBs can be gained from the distributions of $n_{\mathrm{HB}}(r)$, the number of HBs in which a tagged $\mathrm{MeOH}$ molecule located at $r$ participates. Results for $n_{\mathrm{HB}}(r)$ appear in Fig. 4. In the three pores, the profiles at the central regions level off at $\sim 1.85$, a value similar to the one reported for bulk methanol. ${ }^{48}$ Departures from the plateau-like characteristics manifest beyond $r$ $\sim 12 \AA$ in HICs and RHOCs, and somewhat further away, $r \sim 15 \AA$, in HOCs. Note that in the latter case, the drop in the value of $n_{\mathrm{HB}}(r)$ looks much sharper than the one observed in the local density (also shown in the figure), confirming that the single-donor-single-acceptor characteristics are preserved even for those molecules in direct contact with the solid interface. Contrasting, in HICs and RHOCs, the drop in the value of $n_{\mathrm{HB}}(r)$ is much more gradual and goes hand-in-hand with the increment in $\mathrm{HBs}$ involving $\mathrm{Me}-\mathrm{OH}$ and $\mathrm{Si}-\mathrm{OH}$ groups [shaded curves in Figs. 4(b) and 4(c)]. In fact, the $\mathrm{MeOH}$ coordination at $r \sim 14 \AA$ looks practically saturated, i.e., $n_{\mathrm{HB}} \sim 2$. The behavior of the curves adjacent to the interface $(r \gtrsim 15 \AA)$ reveals that the connectivity between $\mathrm{MeOH}$ molecules is practically lost beyond that distance; as such, the outermost peaks of the $n_{\mathrm{HB}}$ profiles cor- 


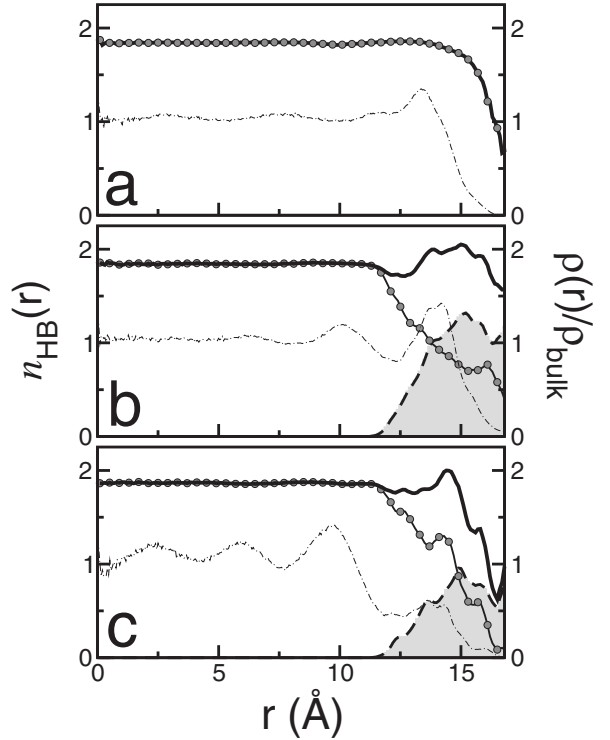

FIG. 4. Average number of H-bonds for methanol-methanol (circles) and methanol-silanol pairs (dashed lines) for (a) HOC, (b) HIC, and (c) RHOC pores. Solid lines correspond to the number of H-bonds between methanol and either surface silanol or another methanol molecule. Methanol local density profiles are also displayed for reference (thin dashed lines, right axes).

respond to a handful of scattered isolated methanol molecules, tightly bounded to silanol groups which lie at surface pockets of difficult access for the solvent.

To complement the previous description, we also examined the energetic characteristics of the $\mathrm{MeOH}$ intermolecular connectivity in the pores by computing the pair energy distributions $P(\varepsilon)$ of the type

$$
P(\varepsilon)=\frac{2}{N_{\mathrm{MeOH}}} \sum_{i}^{N_{\mathrm{MeOH}}} \sum_{j>i}^{N_{\mathrm{MeOH}}}\left\langle\delta\left(\varepsilon_{i j}-\varepsilon\right)\right\rangle,
$$

where $\varepsilon_{i j}$ represents the interaction energy between a pair $i-j$ of $\mathrm{MeOH}$ molecules. The results are shown in Fig. 5. At a qualitative level, the energy distribution for pairs within the pores looks similar to the bulk phase results: ${ }^{30,49}$ apart from the low energy, $\varepsilon \sim 0$, peak characterizing weak interactions between pairs separated by long distances, the profiles exhibit secondary peaks centered at $\sim-23 \mathrm{~kJ} \mathrm{~mol}^{-1}$, arising

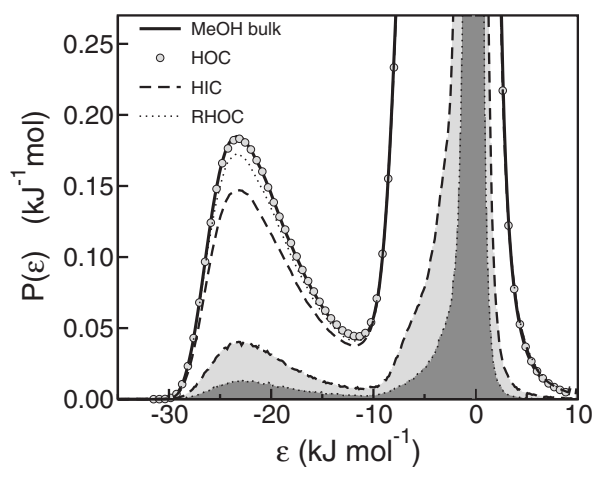

FIG. 5. Pair energy probability distributions for methanol-methanol interactions in bulk (solid line) and confined systems: $\operatorname{HOC}(\bigcirc)$, HIC (-- ), and RHOC $(\cdots)$. The shaded areas correspond to methanol-silanol pair interaction in HIC (gray) and RHOC (dark gray) pores.
TABLE II. Normalized self-diffusion coefficients along different directions for confined methanol (see text).

\begin{tabular}{lcccc}
\hline \hline System & $\tilde{D}_{x}$ & $\tilde{D}_{y}$ & $\tilde{D}_{z}$ & $\tilde{D}$ \\
\hline HOC & 0.41 & 0.40 & 0.51 & 0.45 \\
HIC & 0.31 & 0.30 & 0.38 & 0.33 \\
RHOC & 0.20 & 0.21 & 0.26 & 0.23 \\
\hline \hline
\end{tabular}

from contributions from hydrogen-bonded pairs. In HOCs, the energy distribution at negative values of $\varepsilon$ looks practically identical to that in the bulk phase: no changes are observed neither in the position nor in the height of the HB secondary peak. Interestingly, the energy distribution function in the RHOC pore also looks quite similar, suggesting that most of the $\mathrm{MeOH}$ molecules confined within these much more rugged cavities are able to reconstruct their intermolecular connectivity preserving the number and the strength of the H-bonds. In hydroxylated cavities, while the energy distribution peaks at similar values of $\varepsilon$, the magnitude of the secondary maximum decreases from 0.19 down to 0.14 . This modification confirms the loss of a sizeable number of $\mathrm{MeOH}-\mathrm{MeOH} \mathrm{HBs}$, which turn into $\mathrm{MeOH}-$ $\mathrm{SiOH}$ ones of similar strength. The direct inspection of the low-lying curves also shown in Fig. 5 and representing the intermolecular energy distributions for methanol-silanol pairs in the HIC (dashed lines) and RHOC cavities (dotted lines) confirm the latter assertion: note that the shaded areas practically compensate the differences between the HOC profile and the HIC and RHOC ones.

\section{B. Dynamics}

The translational mobility of methanol within the pores was investigated by computing the self-diffusion coefficient based on the usual Einstein relation,

$$
D=\lim _{t \rightarrow \infty} \frac{1}{2 d t} \mathcal{R}^{2}(t)
$$

where $d$ represents the dimensions of the environment and $\mathcal{R}^{2}(t)$ is the mean-squared displacement in $d$ dimensions,

$$
\mathcal{R}^{2}(t)=\left\langle\left|\mathbf{X}_{i}(t)-\mathbf{X}_{i}(0)\right|^{2}\right\rangle,
$$

with $\left[\mathbf{X}_{i}(t)-\mathbf{X}_{i}(0)\right]$ denoting the projection of the displacement of the center-of-mass of molecule $i$ in $d$ dimensions. Results for different diffusion coefficients normalized by the bulk value $\tilde{D}_{i}=D_{i} / D_{\text {bulk }}\left(D_{\text {bulk }}=2.0 \times 10^{-5} \mathrm{~cm}^{2} \mathrm{~s}^{-1}\right)$ are listed in Table II. Note that in all cases, the overall normalized diffusion coefficients $\widetilde{D}$ are less than half the corresponding bulk value, being the largest retardation the one found in RHOC. Moreover, diffusive motions are clearly anisotropic, being the axial diffusivity $\left(\widetilde{D}_{z}\right) \sim 25 \%-30 \%$ larger than the ones computed along radial directions $\left(\widetilde{D}_{x}, \widetilde{D}_{y}\right)$.

Similarly, the gross features of the rotational dynamics were analyzed in terms of single-dipole autocorrelation function $C(t)$ of the type 


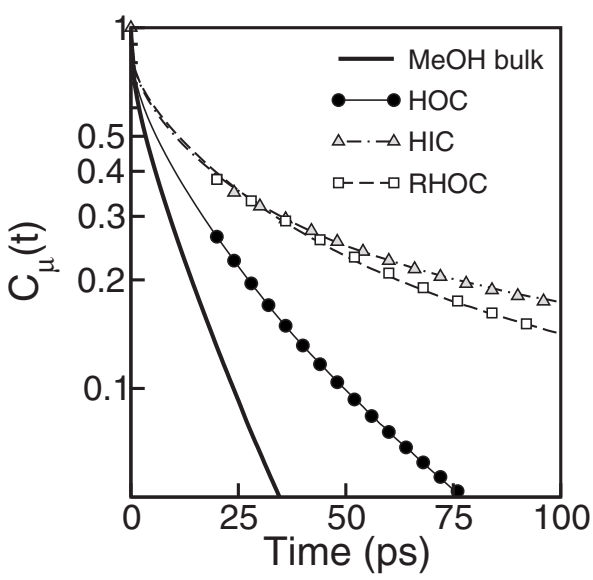

FIG. 6. Single-dipole time correlation function $C_{\mu}(t)$ for bulk and confined methanol. The symbols represent results from the biexponential expression in Eq. (8) for $t>25$ ps.

$$
C_{\mu}(t)=\left\langle\hat{\mu}_{i}(t) \cdot \hat{\mu}_{i}(0)\right\rangle .
$$

In Fig. 6, the orientational correlations $C_{\mu}(t)$ for bulk and confined methanol are displayed. At a first glance, one observes that the temporal decay in the bulk is the fastest, whereas within the pores, one observes the following fast-toslow trend: $\mathrm{HOC} \rightarrow \mathrm{RHOC} \rightarrow \mathrm{HIC}$. From quantitative grounds, these characteristics are also confirmed by average timescales $\left\langle\tau_{\text {rot }}\right\rangle$ obtained from the corresponding time integrals of the four curves (see entries in column 2 of Table III). This trend clearly clashes with the dynamical characteristics of the translational motions in HIC and RHOC, where the slowest behavior was found in the latter cavities. Looking for a physical interpretation of this qualitative difference, we first noted that while the postlibrational curve for bulk $\mathrm{MeOH}$ could be reasonably well described by a single exponential, the long time relaxations for $\mathrm{MeOH}$ within the pores were better described by combinations involving simple and stretched contributions of the type

$$
C(t) \sim a_{1} e^{-t / \tau_{1}}+a_{2} e^{-\left(t / \tau_{2}\right)^{\beta}} \quad t \gg \tau_{\mathrm{lib}},
$$

with $\tau_{2}>\tau_{1}$. Given these characteristics and within the crudest level of approximation, we then speculated on the possibility of adopting a two-state model for $C(t)$. Within this simple scheme, $C_{\mu}(t)$ includes contributions from (i) the central faster molecules relaxing with a timescale $\tau_{1}$ and (ii) a much slower $\mathrm{MeOH}$ shell closer to the pore walls with characteristic orientational decorrelation time $\tau_{2}{ }^{26,35,36}$ Moreover, should this picture be correct, the individual weights $a_{i}$ $(i=1,2)$ should also reflect the relative populations in the central and the external regions obtained from the corresponding integrals of $\rho(r)$. The comparison of the simulated
$C(t)$ and results from nonlinear fitting procedures performed following this line of reasoning are also plotted in Fig. 6, whereas the parameters are listed in columns 3-7 of Table III. Note that the magnitudes of the optimal $\tau_{1}$ and $\tau_{2}$ now reproduce the previous fast-to-slow trend observed for the translational modes. This would confirm that the sole consideration of $\langle\tau\rangle$ might be sometimes misleading, since it includes contributions from slow and fast molecules, with partial weights that depend on the local concentrations of each particular environment under investigation.

The former two-state dynamical model can be further refined by analyzing the translational and the rotational dynamics from a local-property perspective. Following the procedure implemented by Lee et al..$^{50}$ for the analysis of water dynamics surrounding carbohydrates, local diffusion coefficients of $\mathrm{MeOH}, D_{\text {local }}(r)$, were calculated at selected distances $r$ from the pore axis according to the following finitedifference expression:

$$
D_{\text {local }}(r)=\frac{\mathcal{R}_{r}^{2}\left(t_{2}\right)-\mathcal{R}_{r}^{2}\left(t_{1}\right)}{6\left(t_{2}-t_{1}\right)},
$$

where $\mathcal{R}_{r}^{2}(t)$ represents the mean-squared center-of-mass displacement of molecules within cylindrical shells of thickness $\Delta r$ around $r$. Note that the previous expression remains physically sound provided: (i) $t_{1}$ remains longer than the characteristic time required for the individual displacements to reach the usual linear diffusional regime, and at the same time, (ii) $t_{2}$ is sufficiently short so that the tagged $\mathrm{MeOH}$ molecules remain within each cylindrical shell for temporal intervals $\Delta t \sim t_{2}$. For the particular cases investigated here, by setting $\Delta r \sim 1 \AA, t_{1}=2 \mathrm{ps}$, and $t_{2}=3 \mathrm{ps}$, the previous conditions could be reasonably well satisfied. A similar analysis based on individual rotations is straightforward. In this case, local characteristic times $\tau_{\text {local }}(r)$ were obtained from time integrals of local time correlation functions involving the orientational relaxations of molecules lying at $r$ along 3 ps time intervals.

In Fig. 7 the behavior of the local methanol diffusivity $D_{\text {local }}(r)$ and the rotational correlation times $\tau_{\text {local }}(r)$ normalized by the corresponding bulk values are presented. The diffusion coefficients of trapped methanol exhibit a sharp drop as one approaches the pore walls. In all cases, the effects are less marked in HOC and much more marked in RHOC. Results for the hydroxylated pores look intermediate, being closer to the ones for HOCs at the center of the cavities, becoming gradually more comparable to the ones for RHOC near the pore walls. In addition, also note that in RHOCs, important modifications operate in the translational dynamics even at the central part of the pore, where the value

TABLE III. Orientational correlation timescales describing $C_{\mu}(t)$ [see Eqs. (7) and (8)]. Timescales are expressed in ps.

\begin{tabular}{lrccccc}
\hline \hline System & $\left\langle\tau_{\text {rot }}\right\rangle^{\text {a }}$ & $a_{1}$ & $\tau_{1}$ & $a_{2}$ & $\tau_{2}$ & $\beta$ \\
\hline HOC & 17.7 & 0.40 & 15.8 & 0.24 & 46 & 0.94 \\
HIC & 114.0 & 0.32 & 33.7 & 0.22 & 400 & 0.76 \\
RHOC & 90.0 & 0.42 & 39.3 & 0.13 & 550 & 0.95 \\
\hline \hline
\end{tabular}

${ }^{\mathrm{a}}$ Obtained from the time integral of $C_{\mu}(t)$. 

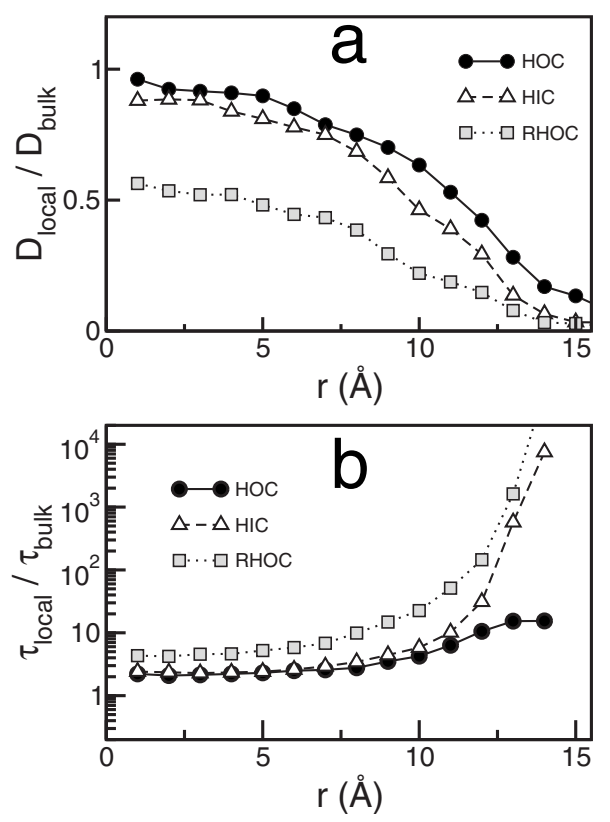

FIG. 7. (a) Average translational diffusion coefficient and (b) average rotational correlation time of methanol as a function of the distance to the axial axis of the cylindrical pore for the three kinds of silica cavities studied.

of $D_{\text {local }}(r)$ is observed to drop down to practically one half of the bulk value. This can be interpreted as a consequence of the propagation of the interface-induced spatial ordering in layers that extend practically down to the central region, as seen in Fig. 2. Yet, confinement effects on the rotational dynamics look more dramatic. In all cases, the mild modifications of $\tau_{\text {local }}(r)$ near the pore axis lead to much sharper increments as $r$ reaches $\sim 10 \AA$. From a quantitative perspective, near hydrophilic and rugged pore walls, the rotational relaxation timescales end up being up to three orders of magnitude slower than the one observed in the bulk.

As such, the disparities between the magnitudes of the retardations operated in the translational and rotational kinetics look akin to the behavior observed in fragile glass formers $^{51,52}$ and in concentrated carbohydrate solutions. ${ }^{53}$ One simple way to monitor the gradual decoupling between the mechanisms that control the different kinetic modes relies on the consideration of simple Brownian models and the Stokes-Einstein-Debye (SED) relations. $^{54}$ Within this scheme, the product between the diffusion coefficient and the characteristic rotational time, namely,

$$
D \tau_{\text {rot }}=\frac{k_{\mathrm{B}} T}{6 \pi \eta r_{\mathrm{hyd}}} \frac{4 \pi r_{\mathrm{hyd}}^{3} \eta}{3 k_{\mathrm{B}} T}=\frac{2}{9} r_{\mathrm{hyd}}^{2},
$$

should remain practically constant. In the previous expression, $\eta$ represents the viscosity of the liquid and $r_{\text {hyd }}$ represents an effective hydrodynamic radius. In fluid media with low and moderate viscosities, both $D$ and $\tau_{\text {rot }}^{-1}$ track the dependence of $T / \eta$ fairly well, whereas near glass transitions and in systems where the dynamical modes are severely hindered, departures from these simple hydrodynamic models manifest in a sensible fashion. In Fig. 8, we present the results illustrating the local behavior of the SED model along the $r$ coordinate. At the central $r \sim 0$ region, predictions of the SED seem to be confirmed by the simulation results.

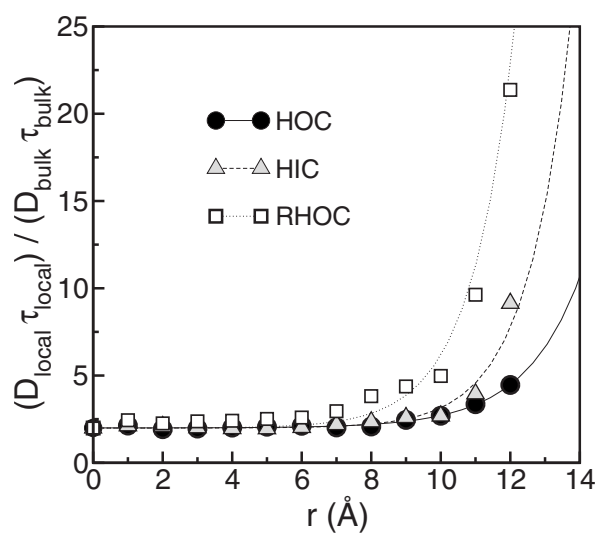

FIG. 8. Product of average translational (local) diffusion coefficient and rotational correlation time of methanol normalized by the corresponding bulk values as a function of the distance to the axial axis in the silica cavity. The curves are guides to the eye.

Departures from the hydrodynamic model start to manifest as one approaches the boundary of the pores, $r \sim 10 \AA$, being the deviations much more marked in the RHOCs.

The final dynamical aspect that we will examine deals with the hydrogen bonding dynamics. Our analysis will be based on the results obtained from the time correlation function of the survival probability of hydrogen bonds of the type

$$
C_{\mathrm{HB}}(t)=\left\langle\eta_{i j}(t) \eta_{i j}(0)\right\rangle
$$

where $\eta_{i j}(t)$ represents the characteristic function that equals 1 if the $i$ - $j$ pair is $\mathrm{H}$-bonded at time $t$, and zero otherwise.

In Fig. 9, we present results for $C_{\mathrm{HB}}(t)$ along with the results from a fitting procedure similar to the one shown in Eq. (8) (see also entries in Table IV for the corresponding parameters). The entries in the last column of Table IV agree with the previous temporal trends observed by the different dynamical modes: compared to the bulk, the differences in the characteristic timescales that dictate the formation and breaking of HBs may stretch up to a factor of $\sim 3$ for the RHOC case. Moreover, from a qualitative perspective, the HB lifetime relaxation in the bulk differs from similar results within the pores. Note that in the former case and in agreement with the previous analysis, ${ }^{49,55}$ the long time decay can be reasonably well described by a single exponential yield-

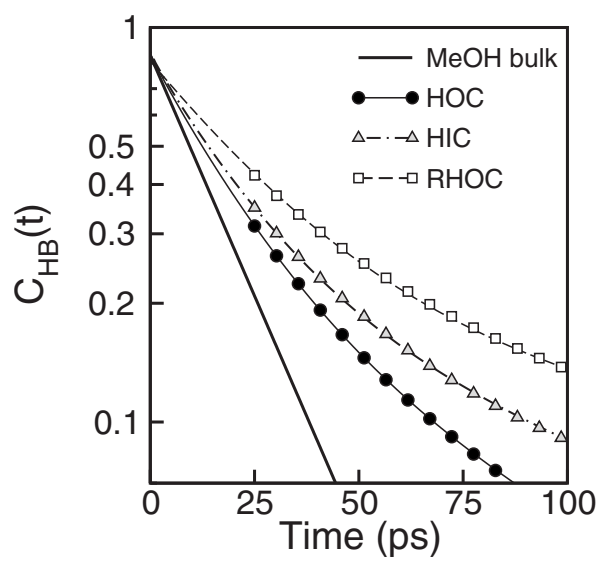

FIG. 9. Time correlation function of hydrogen bonding between methanol molecules, $C_{\mathrm{HB}}(t)$. Same labeling to that shown in Fig. 6. 
TABLE IV. Hydrogen bond lifetime parameters [see Eqs. (11) and (8)]. Times are given in ps.

\begin{tabular}{lcccccc}
\hline \hline System & $a_{1}$ & $\tau_{1}$ & $a_{2}$ & $\tau_{2}$ & $\beta$ & $\left\langle\tau_{\mathrm{HB}}\right\rangle^{\mathrm{a}}$ \\
\hline HOC & 0.53 & 18.4 & 0.33 & 47.3 & 0.77 & 27.6 \\
HIC & 0.53 & 19.7 & 0.37 & 68.6 & 0.73 & 40.9 \\
RHOC & 0.62 & 27.5 & 0.20 & 197 & 0.98 & 56.4 \\
Bulk & 0.85 & 17.8 & $\cdots$ & $\cdots$ & $\cdots$ & 15.20 \\
\hline
\end{tabular}

${ }^{\bar{a}}$ Hydrogen bond lifetime obtained from the time integral of $C_{\mathrm{HB}}(t)$.

ing $\left\langle\tau_{\mathrm{HB}}\right\rangle=15.2 \mathrm{ps}$. Confinement, in turn, modifies the previous characteristics and the relaxations seem to be better described by a combination of two exponential decays. Besides, also note that in HOCs and in HICs, the fastest temporal timescale $\tau_{1}$ is close to the one observed in the bulk; contrasting, in RHOCs, the corresponding relaxation looks different from the two previous scenarios: on the one hand, the values of $\tau_{1}$ increase from $\sim 18-19$ ps up to $\sim 27 \mathrm{ps,}$ while the optimal stretched exponent $\beta$ is practically 1 . A physical interpretation of these differences in terms of modifications in the key mechanisms that control these dynamics is surely called for; however, it largely exceeds the scope of this presentation, so we prefer to postpone it to a forthcoming analysis.

\section{CONCLUDING REMARKS}

The simulation results presented in this paper provide new insights about the structure and the dynamics of liquid methanol confined within three different cylindrical silica pores with nanometric diameters. The first one was a hydrophobic environment, in which solvent-pore interactions were exclusively of the Lennard-Jones type. For these cases, the overall intramolecular connectivity of the liquid, expressed in terms of the number of hydrogen bonds per molecule, suffers from minor modifications. Results for spatial and orientational correlations for the outer solvent shell suggest that the methyl groups mostly lie in contact with the solid substrate.

The second pore environment examined corresponded to cylindrical cavities in which unsaturated oxygen sites at the walls were transformed into polar silanol groups. This introduces additional channels for hydrogen bond formation at the liquid/solid interface and modifies the structure of the nearby liquid in a more sensible fashion. Now, hydroxylic groups in the $\mathrm{MeOH}$ molecules are the ones that remain exposed to the solid substrate, exhibiting a dual HB donoracceptor character.

Lastly, we also analyzed the effects from a larger extent of interface disorder by incorporating bulkier TMS groups at the pore walls. These groups affect the structure of the overall liquid phase as the confinement effects become clearly perceived even at the central regions of the pore. Moreover, the structure of the liquid at outer regions now includes the presence of a sizeable number of isolated molecules, trapped within the surface pockets located between nearby TMS groups, with limited connectivity with the rest of the fluid.

From the dynamical side, the timescales describing translational and rotational motions become significantly stretched, compared to bulk results. Moreover, translational motions become anisotropic, being somewhat more hindered along radial directions than along the axial one. From a qualitative perspective, we observed in all cases the following fast-to-slow trend: $\mathrm{HOC} \rightarrow \mathrm{HIC} \rightarrow \mathrm{RHOC}$. A local dynamical analysis along the radial coordinate of the pore confirms that retardations become increasingly marked near the pore walls and that these effects are much more marked in rotational motions than in translational ones. This disparity and departures from predictions based on the classical Stokes-Einstein-Debye expression suggest a gradual decoupling of the mechanisms that control the different dynamical modes.

In passing, we would like to bring into consideration the experimental evidence that would show trends clashing with some of the previous conclusions. We are referring to the results obtained by Arndt et al. which indicate that TMS groups replacing $\mathrm{OH}$ groups at the surface of porous glasses would act as lubricants, inducing faster orientational relaxations in confined liquid salol. ${ }^{56}$ In the latter case, TMS groups would hinder the formation of H-bonds between the liquid molecules and the substrate, making the orientational motions faster. As a plausible argument to reconcile this apparent inconsistency, we believe that contrasting to the present $\mathrm{MeOH}$ analysis, the linear dimensions of the salol molecules-basically an articulated combination of phenol and salycilic acid moieties - are big enough to prevent solvent trapping between adjacent TMS groups at the surface of the pores. Consequently, predictions on the overall dynamical effects arising from the replacement of $\mathrm{Si}-\mathrm{OH}$ by TMS groups are not straightforward since they are the result of a complex interplay among, at least, four relevant lenghtscales describing (i) the size of the trapped liquid molecules, (ii) the diameter of the pore, (iii) the length of TMS groups, and (iv) the average distance between them at the pore surface.

We are confident that these conclusions will provide elements to achieve an appropriate physical interpretation for more complex phenomena taking place in confined environments, such as, for example, the dynamical analysis of the solvation response of coumarin dyes. Calculations along these lines are being currently undertaken in our laboratory.

\section{ACKNOWLEDGMENTS}

This work was partially funded by ANPCyT (Grant Nos. PICT2007-00300 and PICT2007-00334). M.D.E., J.R., and D.L. are staff members of CONICET (Argentina).

\footnotetext{
${ }^{1}$ S. M. Sharland, Corros. Sci. 27, 289 (1987).

${ }^{2}$ L. W. Lake, Enhanced Oil Recovery (Prentice-Hall, Englewood Cliffs, NJ, 1989).

${ }^{3}$ Y. Wang, C. Bryan, H. Xu, and H. Gao, Geology 31, 387 (2003).
} 
${ }^{4}$ F. Pizzitutti, M. Marchi, F. Sterpone, and P. J. Rossky, J. Phys. Chem. B 111, 7584 (2007).

${ }^{5}$ M. H. H. Pomata, M. T. Sonoda, M. S. Skaf, and M. D. Elola, J. Phys. Chem. B 113, 12999 (2009).

${ }^{6}$ P. Koziol, S. D. Nelson, and J. Jonas, Chem. Phys. Lett. 201, 383 (1993).

${ }^{7}$ J. Zhang and J. Jonas, J. Phys. Chem. 97, 8812 (1993).

${ }^{8}$ J.-P. Korb, L. Malier, F. Cros, S. Xu, and J. Jonas, Phys. Rev. Lett. 77, 2312 (1996).

${ }^{9}$ S. Xu and J. Jonas, J. Phys. Chem. 100, 16242 (1996).

${ }^{10}$ J.-P. Korb, S. Xu, F. Cros, L. Malier, and J. Jonas, J. Chem. Phys. 107, 4044 (1997)

${ }^{11}$ G. Carini, V. Crupi, G. D'Angelo, D. Majolino, P. Migliardo, and Y. B. Melnichenko, J. Chem. Phys. 107, 2292 (1997).

${ }^{12}$ V. Crupi, G. Maisano, D. Majolino, P. Migliardo, and V. Venuti, J. Chem. Phys. 109, 7394 (1998)

${ }^{13}$ D. Morineau, R. Guégan, Y. Xia, and C. Alba-Simionesco, J. Chem. Phys. 121, 1466 (2004)

${ }^{14}$ S. Takahara, S. Kittaka, T. Mori, Y. Kuroda, T. Takamuku, and T. Yamaguchi, J. Phys. Chem. C 112, 14385 (2008).

${ }^{15}$ L. Nikiel, B. Hopkins, and T. W. Zerda, J. Chem. Phys. 94, 7458 (1990).

${ }^{16}$ Y. T. Lee, S. L. Wallen, and J. Jonas, J. Phys. Chem. 96, 7161 (1992).

${ }^{17}$ S. L. Wallen, L. Nikiel, J. Yi, and J. Jonas, J. Phys. Chem. 99, 15421 (1995).

${ }^{18}$ R. Stannarius, F. Kremer, and M. Arndt, Phys. Rev. Lett. 75, 4698 (1995).

${ }^{19}$ A. Huwe, M. Arndt, F. Kremer, C. Haggenmuller, and P. Behrens, J. Chem. Phys. 107, 9699 (1997).

${ }^{20}$ D. Daoukaki, G. Barut, R. Pelster, G. Nimtz, A. Kyritsis, and P. Pissis, Phys. Rev. B 58, 5336 (1998).

${ }^{21}$ J. Banys, M. Kinka, A. Meskauskas, R. Sobiestianskas, W. Volkel, W. Böhlmann, M. Hartmann, and A. Pöppl, Ferroelectrics 346, 173 (2007)

${ }^{22}$ X. Zhu, R. A. Farrer, and J. T. Fourkas, J. Phys. Chem. B 109, 12724 (2005).

${ }^{23}$ C. Streck, Y. B. Mel'nichenko, and R. Richert, Phys. Rev. B 53, 5341 (1996).

${ }^{24}$ B. J. Loughnane and J. T. Fourkas, J. Phys. Chem. B 102, 10288 (1998).

${ }^{25}$ B. J. Loughnane, A. Scodinu, and J. T. Fourkas, J. Phys. Chem. B 103, 6061 (1999)

${ }^{26}$ R. A. Farrer and J. T. Fourkas, Acc. Chem. Res. 36, 605 (2003).

${ }^{27}$ M. Valdéz-González, H. Saint-Martin, J. Hernández-Cobos, R. Ayala, E. Sanchez-Marcos, and I. Ortega-Blake, J. Chem. Phys. 127, 224507 (2007).

${ }^{28}$ T. Yamaguchi, K. Hidaka, and A. K. Soper, Mol. Phys. 96, 1159 (1999).

${ }^{29}$ T. Takamuku, H. Maruyama, S. Kittaka, S. Takahara, and T. Yamaguchi, J. Phys. Chem. B 109, 892 (2005).

${ }^{30}$ R. Guégan, D. Morineau, and C. Alba-Simionesco, Chem. Phys. 317,
236 (2005).

${ }^{31}$ T. Kamijo, A. Yamaguchi, S. Suzuki, N. Teramae, T. Itoh, and T. Ikeda, J. Phys. Chem. A 112, 11535 (2008).

${ }^{32}$ J. Zang, S. Konduri, S. Nair, and D. S. Sholl, ACS Nano 3, 1548 (2009).

${ }^{33}$ V. V. Chaban and O. N. Kalugin, J. Mol. Liq. 145, 145 (2009).

${ }^{34}$ Y. Liu, S. Consta, and W. A. Goddard III, J. Nanosci. Nanotechnol. 10, 3834 (2010).

${ }^{35}$ B. J. Loughnane, R. A. Farrer, and J. T. Fourkas, J. Phys. Chem. B 102, 5409 (1998).

${ }^{36}$ R. A. Farrer, B. J. Loughnane, and J. T. Fourkas, J. Phys. Chem. A 101, 4005 (1997).

${ }^{37}$ J. Rodriguez, M. D. Elola, and D. Laria, J. Phys. Chem. B 114, 7900 (2010).

${ }^{38}$ T. S. Gulmen and W. H. Thompson, Mater. Res. Soc. Symp. Proc. 899E, 0899-N06-05.1.10 (2006).

${ }^{39}$ C. M. Morales and W. H. Thompson, J. Phys. Chem. A 113, 1922 (2009).

${ }^{40}$ T. S. Gulmen and W. H. Thompson, Langmuir 25, 1103 (2009).

${ }^{41}$ A. Brodka and T. W. Zerda, J. Chem. Phys. 95, 3710 (1991).

${ }^{42}$ S. Furukawa, T. Nishiumi, N. Aoyama, T. Nitta, and M. Nakano, J. Chem. Eng. Jpn. 38, 999 (2005).

${ }^{43}$ J. A. D. MacKerell, N. Banavali, and N. Foloppe, Biopolymers 56, 257 (2000).

${ }^{44}$ J. C. Phillips, R. Braun, W. Wang, J. Gumbart, E. Tajkhorshid, E. Villa, C. Chipot, R. D. Skeel, L. Kale, and K. Schulten, J. Comput. Chem. 26, 1781 (2005)

${ }^{45}$ T. A. Darden, D. M. York, and L. G. Pedersen, J. Chem. Phys. 98, 10089 (1993).

${ }^{46}$ U. Essmann, L. Perera, M. L. Berkowitz, T. Darden, H. Lee, and L. G. Pedersen, J. Chem. Phys. 103, 8577 (1995).

${ }^{47}$ M. H. H. Pomata, D. Laria, M. S. Skaf, and M. D. Elola, J. Chem. Phys. 129, 244503 (2008).

${ }^{48}$ M. Matsumoto and K. E. Gubbins, J. Chem. Phys. 93, 1981 (1990).

${ }^{49}$ T. Kosztolányi, I. Bakó, and G. Pálinkás, J. Chem. Phys. 118, 4546 (2003)

${ }^{50}$ S. L. Lee, P. G. Debenedetti, and J. R. Errington, J. Chem. Phys. 122, 204511 (2005).

${ }^{51}$ M. T. Cicerone and M. D. Ediger, J. Chem. Phys. 104, 7210 (1996).

${ }^{52}$ F. H. Stillinger and J. A. Hodgon, Phys. Rev. E 50, 2064 (1994).

${ }^{53}$ M. Rampp, C. Buttersack, and H.-D. Lüdeman, Carbohydr. Res. 328, 561 (2000).

${ }^{54}$ S. R. Becker, P. H. Poole, and F. W. Starr, Phys. Rev. Lett. 97, 055901 (2006).

${ }^{55}$ E. Guàrdia, G. Sesé, and J. A. Padró, J. Mol. Liq. 62, 1 (1994).

${ }^{56}$ M. Arndt, R. Stannarius, H. Groothues, E. Hempel, and F. Kremer, Phys. Rev. Lett. 79, 2077 (1997) 